\title{
Clinical Validation of a Serum Protein Panel (FLNA, FLNB and KRT19) for Diagnosis of Prostate Cancer
}

Shobha Ravipaty ${ }^{1}$, Wenfang Wu${ }^{1}$, Aditee Dalvi ${ }^{1}$, Nikunj Tanna ${ }^{1}$, Joe Andreazi ${ }^{1}$, Tracey Friss ${ }^{1}$, Allison Klotz ${ }^{1}$, Chenchen Liao ${ }^{1}$, Jeonifer Garren ${ }^{1}$, Sally Schofield ${ }^{1}$, Eleftherios P Diamandis ${ }^{2}$, Eric A Klein ${ }^{3}$, Albert Dobi ${ }^{4}$, Shiv Srivastava ${ }^{4}$, Poornima Tekumalla ${ }^{1}$, Michael A Kiebish ${ }^{1}$, Vivek K. Vishnudas ${ }^{1}$, Rangaprasad Sarangarajan ${ }^{1}$, Niven R Narain ${ }^{1}$, Shiv Srivastava ${ }^{4}$ and Viatcheslav R Akmaev ${ }^{1 *}$

${ }^{1}$ Berg, LLC, 500 Old Connecticut Path Framingham, MA 01701, USA

${ }^{2}$ Department of Pathology and Laboratory Medicine, Mount Sinai Hospital, Toronto, Ontario, Canada

${ }^{3}$ Glickman Urological and Kidney Institute, Cleveland Clinic, Cleveland, USA

${ }^{4}$ Department of Surgery, Center for Prostate Disease Research, Uniformed Services University of the Health Sciences and the Walter Reed Military Medical Center, Bethesda, Maryland, USA

\begin{abstract}
This study reports on the development of a novel serum protein panel of three prostate cancer biomarkers, Filamin A, Filamin B and Keratin-19 (FLNA, FLNB and KRT19) using multivariate models for disease screening and prognosis. ELISA and IPMRM (LC-MS/MS) based assays were developed and analytically validated by quantitative measurements of the biomarkers in serum. Retrospectively collected and clinically annotated serum samples with PSA values and Gleason scores were analyzed from subjects who underwent prostate biopsy, and showed no evidence of cancer with or without indication of prostatic hyperplasia, or had a definitive pathology diagnosis of prostatic adenocarcinoma. Probit linear regression models were used to combine the analytes into score functions to address the following clinical questions: does the biomarker test augment PSA for population screening? Can aggressive disease be differentiated from lower risk disease, and can the panel discriminate between prostate cancer and benign prostatic hyperplasia? Modelling of the data showed that the new prostate biomarkers and PSA in combination were better than PSA alone in identifying prostate cancer, improved the prediction of high and low risk disease, and improved prediction of cancer versus benign prostatic hyperplasia.
\end{abstract}

Keywords: Prostate cancer; Prostate specific antigen; Biomarker; FLNA; FLNB; KRT19

Abbreviations: PSA: Prostate Specific Antigen; GS: Gleason Score; FLNA: Filamin-A; FLNB: Filamin-B; KRT19: Keratin-19; IPMRM: Immunoprecipitation Multiple Reaction Monitoring)

\section{Introduction}

Prostate cancer (PrCa) is a leading cause of cancer deaths in American men $[1,2]$. Currently the most prevalent method for detection of PrCa includes screening with a prostate-specific antigen (PSA) blood test followed by a digital rectal examination and diagnostic prostate biopsy. The PSA-based test is first-line for screening of PrCa [3,4]. However, in 2012 the USPTF issued a recommendation against use of PSA-based screening due to its limitations in accuracy. Use of this test has resulted in over-diagnosis and overtreatment of nonlethal cancers, resulting in reduced quality of life of patients who did not require treatment [4]. Thus, there is a need for a diagnostic test with increased predictive power which may reduce the frequency of biopsy, over detection and overtreatment of the $40 \%$ to $50 \%$ of current cases that are indolent [3].

Recent advances in PrCa detection include: tests that measure variants of PSA in blood [5], expression or hypermethylation of PrCa specific genes [6], or proteins in biopsy tissues [7], and expression of $\mathrm{PrCa}$ associated genes [8], or fusion genes in post-digital rectal examination urine [9], or urine exosomes [10]. Markers such as the TMPRSS2-ERG fusion and PCA3 have improved diagnostic accuracy, although discrimination between low risk and more aggressive disease states remains challenging. Indeed, there is a continued need for core biopsy specimens to confirm diagnosis [3].

Therefore, improvements in identification of high-risk cancers from low risk cancers need to be developed when diagnosing prostate cancer, in order to administer the appropriate course of action [10].
High-risk prostate cancers (with a Gleason score of at least 7) have been shown to be more accurately identified using the STHLM3 model, which suggests that structured screening that includes clinical factors, PSA and some PSA derivatives, and germline allelic variants, could reduce the number of prostate biopsies by about a third, when compared to PSA alone [11]. Similarly, a study in 2012 showed that a blood-based Biomarker Panel (CRTAM, CXCR3, FCRL3, KIAA1143, KLF12, TMEM204) could identify men with aggressive prostate cancer, thereby reducing the over-diagnosis and overtreatment that currently results from using PSA alone [12]. These and other studies demonstrate the improvements that have been made in predicting aggressive cancers. Nevertheless, there remains a need for better stratification of patients to low and high-risk forms of the disease.

This study utilizes a novel panel of serum biomarkers to augment the diagnosis of prostate cancer in conjunction with the PSA test. The biomarkers described herein are novel entities and are not PSA derivatives. The panel was developed by using a multiomic approach that defined filamin-A (FLNA), filamin-B (FLNB), and keratin-19 (KRT19) in the panel [13]. New ELISAs for FLNA and FLNB were developed along with immunoprecipitation multiple reaction

*Corresponding author: Viatcheslav R Akmaev, Berg, LLC, 500 Old Connecticut Path, Building B, Framingham, MA 01701, USA, Tel: 6175882235 E-mail: slava.akmaev@berghealth.com

Received January 27, 2017; Accepted February 06, 2017; Published February 08 2017

Citation: Ravipaty S, Wu W, Dalvi A, Tanna N, Andreazi J, et al. (2017) Clinical Validation of a Serum Protein Panel (FLNA, FLNB and KRT19) for Diagnosis of Prostate Cancer. J Mol Biomark Diagn 8: 323. doi: 10.4172/2155-9929.1000323

Copyright: @ 2017 Ravipaty S, et al. This is an open-access article distributed under the terms of the Creative Commons Attribution License, which permits unrestricted use, distribution, and reproduction in any medium, provided the original author and source are credited. 
Citation: Ravipaty S, Wu W, Dalvi A, Tanna N, Andreazi J, et al. (2017) Clinical Validation of a Serum Protein Panel (FLNA, FLNB and KRT19) for Diagnosis of Prostate Cancer. J Mol Biomark Diagn 8: 323. doi: 10.4172/2155-9929.1000323

monitoring (IPMRM) for FLNA, which resulted in significant improvements in the context of PrCa detection and prognosis.

\section{Materials and Methods}

\section{Human and animal rights}

All procedures performed in studies involving human participants were in accordance with the ethical standards of the institutional and/ or national research committee and with the 1964 Helsinki declaration and its later amendments or comparable ethical standards. All applicable international, national, and/or institutional guidelines for the care and use of animals were followed.

\section{Quantitation of FLNA in human serum samples by ELISA}

Antibodies against FLNA were developed by immunizing mice with E. coli-expressed partial FLNA protein (aa 1443-2131). Hybridomas were selected by affinity to HEK293-expressed full length FLNA by ELISA and BLI (Bio-layer interferometry). Antibodies were further screened to ascertain no reactivity to Filamin family members FLNB and FLNC. Sandwich ELISA was optimized by R\&D Systems using two high affinity FLNA antibodies (3F4 and 6E3 developed by Berg, LLC in-house) and HEK293-expressed full length FLNA as a calibrator.

\section{Quantitation of FLNB in human serum samples by ELISA}

Antibodies against FLNB were developed by immunizing mice with E. coli-expressed partial FLNB protein (aa 1416-2089). Hybridomas were selected by affinity to HEK293-expressed full length FLNB by ELISA and BLI (Bio-layer interferometry). Antibodies were further screened to ensure no cross-reactivity to the other two filamin family members, FLNA and FLNC. Sandwich ELISA was optimized by R\&D Systems using two high affinity FLNB antibodies (3F10 and 5 H7 produced in-house) and HEK293-expressed full length FLNB as calibrator.

\section{FLNA and FLNB assay validation}

The following parameters were assessed during assay validation: Calibration curve precision and accuracy were evaluated using 4-PL non-linear regression model over 6 assay runs. Intra and inter-run QC precision was evaluated over 6 separate daily runs using both lyophilized QC's and matrix QC's $(\mathrm{n} \geq 34)$. Short-term stability was evaluated for up to 24 hours at $4^{\circ} \mathrm{C}$ (FLNA), 6 hours at $4^{\circ} \mathrm{C}$, and 4 hours at benchtop (FLNB). Long-term stability was assessed using serum samples stored at $-80^{\circ} \mathrm{C}$. Spike recovery and dilution linearity $(8$-fold) were evaluated throughout the assay working range. Freeze-thaw stability of up to three cycles was tested at $-80^{\circ} \mathrm{C}$. Potential interferences were evaluated by spiking samples with hemoglobin $(50 \mathrm{mg} / \mathrm{dL})$, unconjugated bilirubin $(3 \mathrm{mg} / \mathrm{dL})$, and triglyceride-rich lipoproteins $(2170 \mathrm{mg} / \mathrm{dL})$. Cross-reactivity was evaluated using FLNB recombinant protein on FLNA assay and plate homogeneity was evaluated using spiked matrix.

\section{Quantitation of KRT19 in human serum samples by ELISA}

KRT19 was assessed using a commercially available diagnostic ELISA kit per manufacturer's instructions (Fujirebio, Malvern, PA). This kit detects the CYFRA-21-1 fragment of KRT19. Manufacturer's instructions were followed for sample testing.

\section{KRT19 assay validation}

The following parameters were assessed during assay validation: Calibration curve precision and accuracy were evaluated using suggested 4-degree polynomial regression model over 5 assay runs.
Intra and inter-run QC precision was verified over 6 separate daily runs using both lyophilized QC's and serum QC's $(\mathrm{n} \geq 12)$. Shortterm stability was evaluated for up to 24 hours at $4^{\circ} \mathrm{C}$, benchtop and $37^{\circ} \mathrm{C}$. Freeze-thaw stability was assessed for up to five cycles at $-80^{\circ} \mathrm{C}$. Spike recovery was evaluated at $\mathrm{mid}(25 \mathrm{ng} / \mathrm{ml})$ and high $(50 \mathrm{ng} / \mathrm{ml})$ analytical range of the assay.

\section{Quantitation of FLNA peptides by immunoprecipitation and LC-MS/MS (MRM) analysis}

Antibody immobilization: Three mouse monoclonal antibodies, Anti-FLNA 2C12, Anti-FLNA 3F4, and Anti-FLNA 6E3 (Berg, as used in the ELISA described above) were immobilized onto an agarose support using the ThermoFisher Scientific Pierce Direct IP Kit (ThermoFisher Scientific) according to the manufacturer's protocol with a few modifications. $200 \mu \mathrm{g}$ of each of the three antibodies, were coupled individually to $200 \mu \mathrm{L}$ of AminoLink Plus coupling resin and stored at $4^{\circ} \mathrm{C}$ until needed.

Immunoprecipitation and preparation of calibration standards: Immunoprecipitation was performed using the Pierce Direct IP Kit (ThermoFisher Scientific) according to the manufacturer's protocol with few modifications. Immunoprecipitation tubes were prepared by aliquoting $5 \mu \mathrm{L}$ of each of the three antibody-coupled resins into the IP tube (Pierce Direct IP Kit, ThermoFisher Scientific). The resin was washed twice with $200 \mu \mathrm{L}$ of IP lysis/wash buffer. $100 \mu \mathrm{L}$ of human serum sample or $100 \mu \mathrm{L}$ of water (surrogate matrix) was added to each IP tube along with $500 \mu \mathrm{L}$ of prepared lysis buffer solution (IP lysis/wash buffer with 1.2X Halt protease cocktail inhibitor (ThermoFisher Scientific) and $0.5 \mathrm{M}$ EDTA and incubated overnight at $4^{\circ} \mathrm{C}$ with end-over-end mixing. The resin was washed five times with $200 \mu \mathrm{L}$ of IP lysis/wash buffer and once with $100 \mu \mathrm{L}$ of $1 \mathrm{X}$ conditioning buffer. The captured proteins were eluted with $50 \mu \mathrm{L}$ of elution buffer with an incubation time of 15 minutes and neutralized with $5 \mu \mathrm{L}$ of $1 \mathrm{M}$ Tris $\mathrm{HCl}, \mathrm{pH} 9.0$ (Teknova, Hollister, CA). The IP eluates from the surrogate matrix were used to prepare P2 (AGVAPLQV) and P4 (YNEQHVPGSPFTA) peptide calibration curves by spiking with a $\mathrm{P} 2 / \mathrm{P} 4$ synthetic peptide (Genscript, Piscataway, NJ) stock solution $(0.2 / 0.36 \mu \mathrm{g} / \mathrm{mL})$ followed by serial dilution. $\mathrm{P} 2$ and $\mathrm{P} 4$ calibration standards ranged from $125 \mathrm{pg} /$ $\mathrm{mL}$ to $2000 \mathrm{pg} / \mathrm{mL}$ and $1125 \mathrm{pg} / \mathrm{mL}$ to $36000 \mathrm{pg} / \mathrm{mL}$, respectively. All samples were then subjected to trypsin digestion as described below.

Digestion of IP extracted samples using trypsin: Trypsin digestion was performed using the Flash Digest Kit (Perfinity Biosciences, West Lafayette, IN) following the manufacturer's protocol with few modifications. Flash digest tubes were equilibrated to room temperature and then centrifuged for $1 \mathrm{~min}$ at $1500 \times \mathrm{g}$ and $5^{\circ} \mathrm{C} .50$ $\mu \mathrm{L}$ of each sample, $25 \mu \mathrm{L}$ of digestion buffer (Perfinity Biosciences), and $5 \mu \mathrm{L}$ of working internal standard (ThermoFisher Scientific) solution (P2/P4 10/30 ng/mL) were added to the Flash digest tubes. After vortexing, samples were digested at $70^{\circ} \mathrm{C}$ for 20 minutes in the Eppendorf, ThermoMixer $\mathrm{C}$ (Eppendorf). The Flash digest tubes were then centrifuged for 5 minutes at $1500 \times \mathrm{g}$ and $5^{\circ} \mathrm{C}$. A $60 \mu \mathrm{L}$ aliquot of the supernatant was transferred to an LC-MS vial.

LC-MS/MS (MRM) analysis: MRM analyses were performed on a 6500 QTRAP mass spectrometer (Sciex) equipped with an electrospray source, a 1290 Infinity UPLC system (Agilent Technologies, Santa Clara, CA) and a XBridge Peptide BEH300 C18 $(3.5 \mu \mathrm{m}, 2.1 \mathrm{~mm} \times$ $150 \mathrm{~mm}$ ) column (Waters, Milford, MA). Liquid chromatography was carried out at a flow rate of $400 \mu \mathrm{L} / \mathrm{min}$, and the sample injection volume was $30 \mu \mathrm{L}$. The column was maintained at a temperature of $60^{\circ} \mathrm{C}$. Mobile phase A consisted of $0.1 \%$ formic acid (Sigma Aldrich) in 
Citation: Ravipaty S, Wu W, Dalvi A, Tanna N, Andreazi J, et al. (2017) Clinical Validation of a Serum Protein Panel (FLNA, FLNB and KRT19) for Diagnosis of Prostate Cancer. J Mol Biomark Diagn 8: 323. doi: 10.4172/2155-9929.1000323

water (ThermoFisher Scientific) and mobile phase B consisted of $0.1 \%$ formic acid in acetonitrile (ThermoFisher Scientific). The gradient with respect to \% $\mathrm{B}$ was as follows: 0 to $1.5 \mathrm{~min}, 5 \%$; 1.5 to $2 \mathrm{~min}, 5 \%$ to $15 \%$; 2 to $5 \mathrm{~min}, 15 \%$; 5 to $7.1 \mathrm{~min}, 15 \%$ to $20 \%$; 7.1 to $8.1 \mathrm{~min}, 20 \%$ to $80 \%$; 8.1 to $9.0 \mathrm{~min}, 80 \%$; and 9.0 to $9.1 \mathrm{~min}, 80 \%$ to $5 \% .9 .1$ to $16 \mathrm{~min}$, $5 \%$. The instrument parameters for 6500 QTRAP mass spectrometer were as follows: Ion spray voltage of $5500 \mathrm{~V}$, curtain gas of $20 \mathrm{psi}$, collision gas set to "medium", interface heater temperature of $400^{\circ} \mathrm{C}$, nebulizer gas (GS1) of 80 psi and ion source gas (GS2) of 80 psi and unit resolution for both Q1 and Q3 quadrupoles.

Selection of surrogate peptides and MRM transitions: Potential surrogate peptides for FLNA quantitation were initially chosen by Skyline software [14] and LC-MS/MS analysis (LTQ Orbitrap Velos coupled to Eksigent nano-LC) of recombinant FLNA protein (GenScript) tryptic digest. From the list of potential surrogate peptides, two surrogate peptides, peptide 2 (AGVAPLQVK) and peptide 4 (YNEQHVPGSPFTAR) were selected based on surrogate peptide selection rules [15] and signal intensities of the peptides in spiked and unspiked serum digests. The uniqueness of the surrogate peptides to the target protein was confirmed by running BLAST searches. Heavy labeled versions of the surrogate peptide 2 and 4, AGVAPLQV [K(13C6; $15 \mathrm{~N} 2)]$ and NEQHVPGSPFTA[R(13C6; 15N4)] were used as internal standards. MRM transitions were optimized using synthetic surrogate peptides (GenScript) and their internal standards (ThermoFisher Scientific) and the following $\mathrm{m} / \mathrm{z}$ transitions were monitored: $\mathrm{P} 2,441.7$ $(\mathrm{M}+2 \mathrm{H})^{2+} \rightarrow 584.5\left(\mathrm{y}_{5}{ }^{1+}\right)$; P4 $535(\mathrm{M}+3 \mathrm{H})^{3+} \rightarrow 832.4\left(\mathrm{y}_{8}{ }^{1+}\right)$, P2_IS 445.5 $(\mathrm{M}+2 \mathrm{H})^{2+} \rightarrow 592.1\left(\mathrm{y}_{5}{ }^{1+}\right)$; P4_IS, $538.4(\mathrm{M}+3 \mathrm{H})^{3+} \rightarrow 842.5\left(\mathrm{y}_{8}{ }^{1+}\right)$.

\section{IPMRM data analysis and quantitation}

Data analysis was performed using the Analyst ${ }^{\circledR}$ software (version 1.6.2, AB Sciex, Framingham, MA) and peak integrations were reviewed manually. The calibration curve for FLNA P2 and P4 peptides was constructed by plotting the peak area ratios (analyte/ internal standard) versus concentration of the standard with $1 / \mathrm{x}^{2}$ linear least square regression. The regression equations from P2 and P4 calibration standards was used to back-calculate the measured P2 and $\mathrm{P} 4$ concentrations for each QC and unknown sample.

\section{Assay validation}

The following parameters were assessed during assay validation: Calibration curve linearity and linearity regression weighting factor were assessed from 4 independent calibration curves for P2 and P4. Intra and inter-batch precision of the assay was evaluated by analyzing low QC (LQC) and high QC (HQC) human serum samples (6 replicates each) on different days. LQC and HQC samples were also used to assess sample stability in the autosampler $\left(4^{\circ} \mathrm{C}\right.$ stored up to 48 hours), short term stability (at $4^{\circ} \mathrm{C}$ and ambient temperature, stored up to 48 hours), long term stability (at $-80^{\circ} \mathrm{C}$ ) and freeze-thaw stability ( up to three cycles at $-80^{\circ} \mathrm{C}$ and $-20^{\circ} \mathrm{C}$ ), post-preparation stability (at $-20^{\circ} \mathrm{C}$ ). Potential interferences were evaluated by spiking HQC and LQC samples with hemoglobin $(500 \mathrm{mg} / \mathrm{dL})$, unconjugated bilirubin $(30 \mathrm{mg} / \mathrm{dL})$, and triglycerides $(1000 \mathrm{mg} / \mathrm{dL})$. In addition, carry over and instrument drift were also assessed.

\section{Sample collection}

Clinically annotated serum samples with PSA values and Gleason scores (GS) were collected from males visiting Mount Sinai Hospital, Toronto, Canada between September 2007 and April 2008 with prostatic symptoms. Samples were collected just before biopsy from 662 patients who underwent prostatic biopsy that resulted in definitive diagnosis of prostatic conditions including prostate cancer $(\mathrm{n}=311)$, benign conditions $(n=122)$, atypical small acinar proliferation $(n=26)$, inflammation $(n=58)$, prostatic intraepithelial neoplasia $(n=69)$, microfocus adenocarcinoma $(n=16)$, and benign prostatic hyperplasia $(n=60)$. A total of 503 samples were included in the final analysis; 159 samples were removed from analysis due to missing variable values in either FLNA, FLNB, P2, or P4.

\section{Statistical analysis}

Regression models were built and compared for their ability to classify patients with prostate cancer with low GS $(\leq 6)$, high GS $(\geq 7)$, and with an absence of cancer on biopsy. The resulting Prostate Cancer Panel predictive algorithms were based on the regression models and probability threshold values selected to achieve a certain level of test sensitivity or specificity. All analyses were performed in R 3.2.2 with significance level of 0.05 , unless otherwise stated.

\section{Results}

This study describes the development of novel biomarker assays to screen for and monitor prostate cancer in concert with the existing PSA test. Two ELISAs were developed for the new biomarkers FLNA and FLNB. The existing commercial assays were found to perform suboptimally. The other biomarker in the panel, KRT19, already had a commercially available ELISA kit for use. Our preliminary investigations on the quantitation of FLNA, FLNB and KRT19 using an MRM-only approach proved to be futile due to insufficient sensitivity. Since our novel biomarkers were low abundance proteins in human plasma/serum matrices, development of quantitative mass spectrometry based assays was particularly challenging. To improve the sensitivity of the assay, an immunoaffinity enrichment approach coupled with MRM was evaluated using the antibodies developed for ELISA. Of the three biomarkers, an IPMRM assay was feasible only for FLNA biomarker in serum matrix.

\section{FLNA, FLNB and KRT19 ELISA validation}

FLNA and FLNB ELISA method development involved optimization of several parameters including antibody pair selection, blocking buffers, assay diluents, incubation times, matrix selection, selectivity and sensitivity. The assay performance was then evaluated by performing a validation study in the serum matrix. Since the KRT19 assay was a commercial kit, validation of the assay was performed inhouse. All ELISA assays met validation acceptance criteria as detailed in Table 1.

\section{FLNA IPMRM assay development and validation}

FLNA IPMRM assay development involved optimization of several parameters including: selection of antibodies, immobilization, immunoaffinity capture, incubation, elution, trypsin digestion and other mass spectrometry parameters. The performance of the FLNA IPMRM assay was assessed using serum QCs and the assay met validation acceptance criteria as shown in Supplementary Table 1.

\section{Comparison of the Prostate Cancer Biomarker Panel FLNA, FLNB and KRT19, versus PSA alone for prediction of prostate cancer}

Sera from patients were then tested with the biomarker ELISAs and IPMRM. The results were combined with data on age, PSA, and Gleason score and subjected to regression modelling. Table 2 shows the patient demographic data pertaining to age, cancer status, GS and benign classification for the samples analyzed in this study. The Prostate Cancer Biomarker Panel, (biomarkers FLNA, FLNB, age 
Citation: Ravipaty S, Wu W, Dalvi A, Tanna N, Andreazi J, et al. (2017) Clinical Validation of a Serum Protein Panel (FLNA, FLNB and KRT19) for Diagnosis of Prostate Cancer. J Mol Biomark Diagn 8: 323. doi: 10.4172/2155-9929.1000323

Page 4 of 8

\begin{tabular}{|c|c|c|c|}
\hline Study & FLNA & FLNB & KRT19 \\
\hline Analytical Range & $3.13 \mathrm{ng} / \mathrm{ml}$ to $200 \mathrm{ng} / \mathrm{ml}$ & $0.087 \mathrm{ng} / \mathrm{ml}$ to $2.79 \mathrm{ng} / \mathrm{ml}$ & $0.5 \mathrm{ng} / \mathrm{ml}$ to $50 \mathrm{ng} / \mathrm{ml}$ \\
\hline $\mathrm{R}^{2}$ of calibration curves & $\geq 0.99$ & $\geq 0.99$ & $\geq 0.99$ \\
\hline Intra-day Precision & $C V<10 \%(n=8)$ & $C V<14.6 \%(n=5)$ & $C V<6.7 \%(n=8)$ \\
\hline Inter-day Precision & $C V<8.7 \%(n=41)$ & $C V<23 \%(n=34)$ & $C V<19.2 \%(n=15)$ \\
\hline Spike Recovery in serum & $124 \%$ & $89 \%$ & $98-121 \%$ \\
\hline Dilutional Linearity in serum & $\%$ bias $<20 \%$ for up to $1: 8$ dilution & $\%$ bias $<20 \%$ for up to $1: 8$ dilution & N/A \\
\hline Freeze-Thaw Stability in serum & Stable up to 3 freeze-thaw cycles & Stable up to 3 freeze-thaw cycles & Stable up to 5 freeze-thaw cycles \\
\hline Short-term Stability in serum & $\begin{array}{c}\text { Stable for } 2 \text { hours at room temperature and } \\
\text { at } 6 \text { hours at } 4^{\circ} \mathrm{C}\end{array}$ & $\begin{array}{c}\text { Stable for } 4 \text { hours at room temperature } \\
\text { and } 6 \text { hours at } 4^{\circ} \mathrm{C}\end{array}$ & $\begin{array}{l}\text { Stable for } 4 \text { hours at room } \\
\text { temperature and } 24 \text { hours at } 4^{\circ} \mathrm{C}\end{array}$ \\
\hline Long-term Stability in serum & Stable for up to 1 year at $-80^{\circ} \mathrm{C}$ & Stable for up to 1 year at $-80^{\circ} \mathrm{C}$ & $\mathrm{N} / \mathrm{A}$ \\
\hline Interfering Substances in serum & $\begin{array}{l}\text { No interference for levels below } \\
250 \text { mg/dL Hemoglobin; } \\
30 \text { mg/dL Bilirubin; } \\
1000 \text { mg/dL Lipoproteins }\end{array}$ & $\begin{array}{l}\text { No interference for levels below } \\
50 \text { mg/dL Hemoglobin; } \\
3 \text { mg/dL Bilirubin; } \\
2170 \text { mg/dL Lipoproteins }\end{array}$ & N/A \\
\hline Specificity in serum & $\begin{array}{l}\text { No cross reactivity with FLNB protein at } 10 \\
\text { pM }\end{array}$ & $\begin{array}{l}\text { No cross reactivity with FLNA protein at } \\
\qquad 179 \mathrm{pM}\end{array}$ & $\mathrm{N} / \mathrm{A}$ \\
\hline
\end{tabular}

Table 1: FLNA, FLNB and KRT19 ELISA validation summary. All levels reported in this table are within the appropriate acceptance criteria.

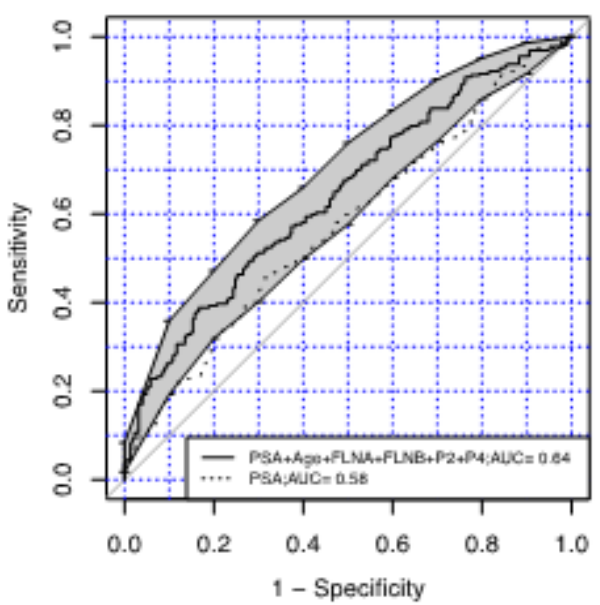

c

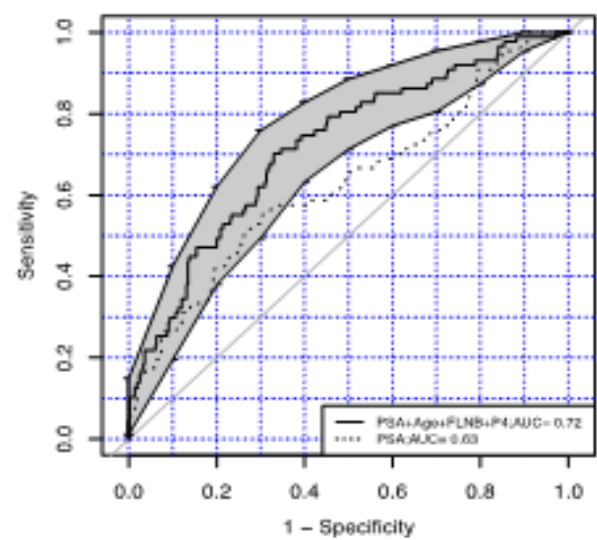

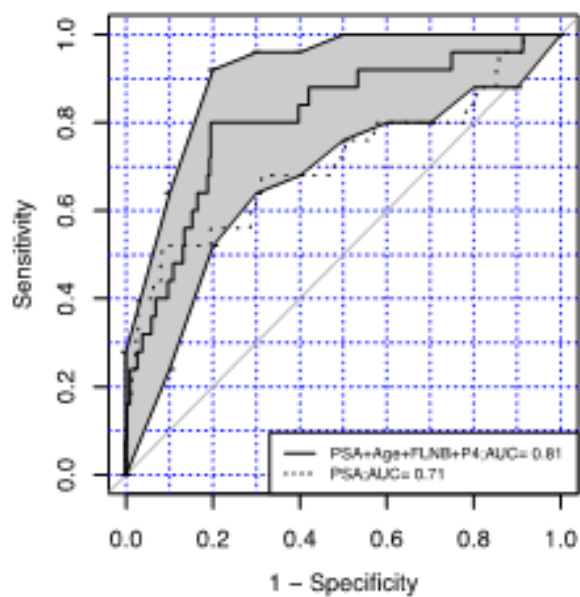

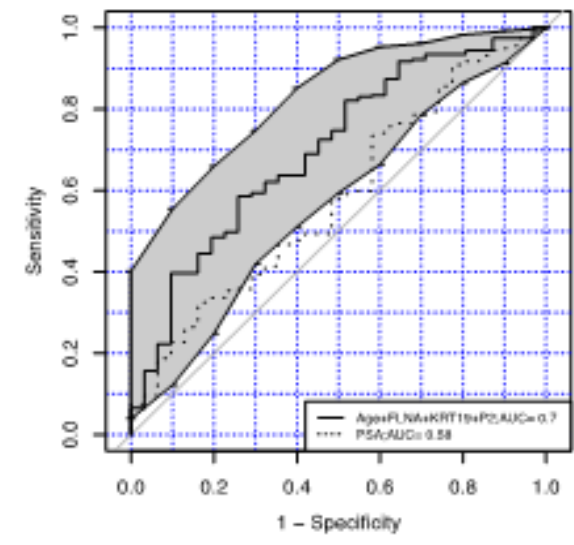

Figure 1: ROC curves of regression models using the prostate biomarker panel, age and PSA test compared to PSA alone. 1A) Prostate Biomarker Panel (FLNA, FLNB, age and PSA) predicts prostate cancer more accurately than PSA alone between patients with or without prostate cancer (Prostate Biomarker Panel AUC, $0.64(0.59,0.69)$, PSA alone AUC, 0.58). 1B) Prostate biomarker FLNB, Age and PSA discriminates between patients with either Gleason $\leq 6$, or Gleason $\geq 7$, over use of PSA alone (Prostate panel AUC, 0.81, (0.71, 0.9), PSA alone AUC, 0.71). 1C) Prostate biomarker FLNB, Age, PSA and low Gleason score ( $\leq 6)$ predicts likelihood of low-risk disease over use of PSA alone (Prostate panel AUC, $0.72(0.66,0.78)$, PSA alone AUC, 0.63). 1D) Prostate biomarkers FLNA, KRT19 and Age with PSA discriminates between prostate cancer and benign prostatic hyperplasia over use of PSA alone (Prostate panel AUC, 0.70, (0.60, 0.80), PSA alone AUC, 0.58).

and PSA) improved the classification of prediction of prostate cancer over PSA alone (AUC=0.64, $[0.59,0.69]$, vs 0.58) (Figure $1 \mathrm{~A})$. The predictive algorithm was set to have a cutoff $=0.45$, which is based on the regression model achieving sensitivity equivalent to PSA=4 ng/ $\mathrm{ml}$. The distribution of predicted probabilities for patients with and without PrCa are shown in Figure 2A. 
Citation: Ravipaty S, Wu W, Dalvi A, Tanna N, Andreazi J, et al. (2017) Clinical Validation of a Serum Protein Panel (FLNA, FLNB and KRT19) for Diagnosis of Prostate Cancer. J Mol Biomark Diagn 8: 323. doi: 10.4172/2155-9929.1000323

A

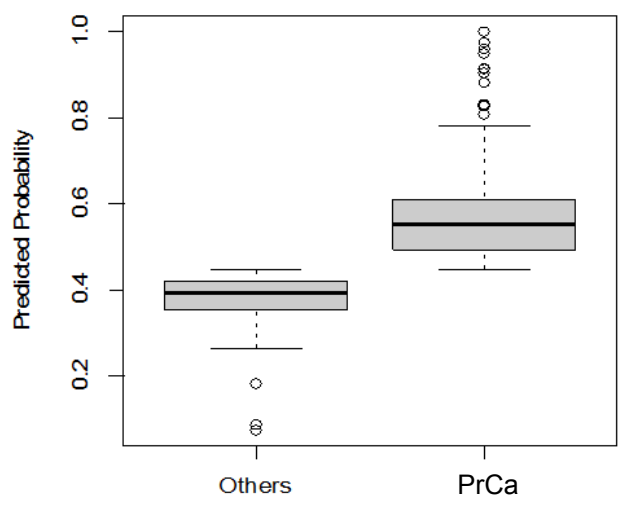

C

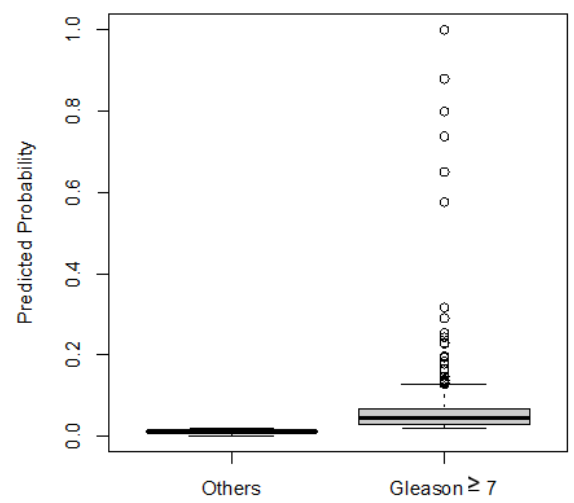

B

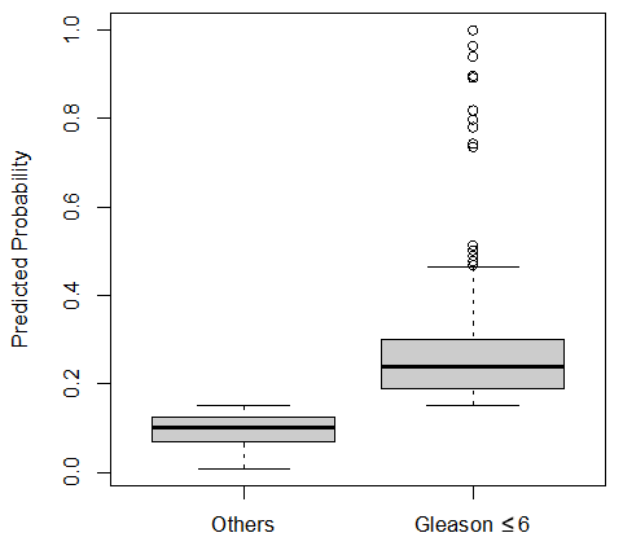

D

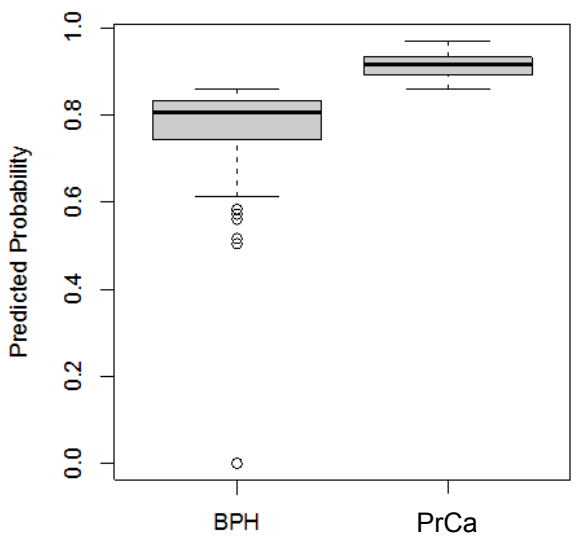

Figure 2: Predicted probability distributions plotted for each ROC analysis. 1A) Distribution of predicted probabilities for patients with or without PrCa. 1B) Distribution of predicted probabilities for patients with high Gleason score (GS $\geq 7$ ) disease. 1C) Distribution of predicted probabilities for patients with Gleason score $\leq$ 6. 1D) Distribution of predicted probabilities for patients with benign prostatic hyperplasia or PrCa.

High-risk disease prediction with FLNB, age and PSA, compared with PSA alone

Comparing serum samples from patients with high GS $(\geq 7)$ and samples from patients with low GS $(\leq 6)$ with our biomarker FNLB, age and PSA, against the use of PSA alone is shown in Figure 1B. The model that achieved the greatest prediction between patients with GS $\leq 6$ and patients with GS $\geq 7$ is a regression model with the biomarker FLNB, age and PSA. The algorithm was set to have a cutoff $=0.02$, which is based on the regression model achieving sensitivity $\geq 0.95$. When compared with PSA alone, regression modelling with FLNB, age and PSA improved the classification of low and high Gleason scores $(\mathrm{AUC}=0.81[0.70,0.90]$, vs 0.71 ). The distribution of predicted probabilities for patients with Gleason score $\leq 6$ and Gleason score $\geq 7$ are shown in a box plot (Figure 2B).

Low-risk disease prediction with FLNA, age and PSA, compared with PSA alone

Patient serum samples with Gleason scores $\leq 6$ were analyzed with our biomarker FLNA, age and PSA, compared with PSA alone as shown in Figure 1C. The model that achieved the highest prediction for patients with low Gleason score $(\leq 6)$ over PSA alone is a regression model with biomarker FLNB, age and PSA vs PSA alone. The predictive algorithm was set to have a cutoff $=0.15$, which is based on the regression model achieving a sensitivity of $\geq 0.8$. The Biomarker Panel FLNB, age and PSA has improved classification over PSA alone (AUC 0.72 [0.66, 0.78 ] vs 0.63$)$. The distribution of predicted probabilities for patients with low Gleason score are shown in a boxplot in Figure 2C.

Prediction of benign prostatic hyperplasia versus prostate cancer with FLNA, KRT19 and age, compared with PSA alone

Samples of patient sera were analyzed with the biomarkers FLNA, KRT19 and age combined, versus PSA alone. Figure 1D showed the highest prediction between patients with benign prostatic hyperplasia versus PrCa. The predictive algorithm was set to have a cutoff $=0.86$, which is based on the regression model achieving sensitivity $\geq 0.8$. The biomarkers FLNA, KRT19 and age have improved classification over PSA alone (AUC $=0.70[0.60,0.80]$, vs 0.58 ). The distribution of predicted probabilities for patients with benign prostatic hyperplasia versus PrCa is shown in Figure 2D.

Supplementary Table 2 summarizes the cutoff, AUC, sensitivity, specificity, positive predictive values (ppv), and negative predictive values (npv) of the predictive algorithms for each comparison.

\section{Discussion}

This manuscript describes the validation of a novel Biomarker Panel for prostate cancer screening using traditional ELISA and IPMRM for analysis of patient serum samples. IPMRM combines IP with mass spectrometry and allows the rapid quantitation of proteins with 


\begin{tabular}{|c|c|c|c|}
\hline & Age Range & Mean & SD \\
\hline BPH & $48-75$ & 59 & 11 \\
\hline Benign & $45-82$ & 61 & 7 \\
\hline Gleason $\leq 6$ & $42-84$ & 62 & 7 \\
\hline Gleason $=7$ & $45-82$ & 65 & 8 \\
\hline Gleason $>7$ & $52-83$ & 68 & 7 \\
\hline \multicolumn{4}{|c|}{ Cancer Classification } \\
\hline Benign & 224 & & \\
\hline Cancer & 279 & & \\
\hline Total & 503 & & \\
\hline \multicolumn{4}{|c|}{ Gleason Score } \\
\hline$<6$ & 2 & & \\
\hline 6 & 156 & & \\
\hline 7 & 74 & & \\
\hline$\geq 8$ & 28 & & \\
\hline Gleason score N/A & 243 & & \\
\hline Total & 503 & & \\
\hline \multicolumn{4}{|c|}{ Benign Classification } \\
\hline Benign & 81 & & \\
\hline $\mathrm{N} / \mathrm{A}$ & 6 & & \\
\hline $\begin{array}{l}\text { Other-Benign prostatic } \\
\text { hyperplasia }\end{array}$ & 34 & & \\
\hline Other-Inflammation & 46 & & \\
\hline $\begin{array}{l}\text { Other-Prostatic Intraepithelial } \\
\text { Neoplasia }\end{array}$ & 57 & & \\
\hline Total & 224 & & \\
\hline
\end{tabular}

Note: Patient demographic data for the samples in this study. Table shows number of benign and cancer cases, Gleason scores and benign classification breakdown for the population studied.

Table 2: Patient demographic data for the samples in this study. Table shows number of benign and cancer cases, Gleason scores and benign classification breakdown for the population studied.

enhanced sensitivity and specificity. For biomarkers, this technique has shown to achieve low $\mathrm{ng} / \mathrm{mL}$ quantitation by selective enrichment of target proteins in complex matrices [16-18].

Currently, there is an unmet clinical need for a more specific and accurate test for prostate cancer. The standard of care for prostate cancer diagnosis is the PSA test in combination with diagnostic prostate biopsy. However, the PSA test has a high false positive rate and may not reflect true cancer detection. In 2012, the US Preventive Services Task Force issued a recommendation against the use of PSA screening due to the over-detection and overtreatment of non-lethal cancers [3]. To confirm diagnosis, patients undergo invasive prostate biopsies that may cause infection and urinary dysfunction [19].

Continued use of the PSA test and prostate biopsy places a heavy burden on the patient and healthcare system with over-diagnosis, unnecessary biopsies and increased costs. From 2006-2009, Medicare spent $\$ 450$ million annually on PSA screening and subsequent diagnostic procedures. Additionally, the cost of screening men over 75 years, the population least likely to benefit from the PSA test, was $\$ 145$ million annually during this time period, representing a third of total Medicare spending on prostate cancer screening [20]. Current efforts focus on the development of non-invasive biomarkers to distinguish between $\mathrm{PrCa}$ and benign prostatic hyperplasia, aggressive and indolent forms of the disease, with the aim of reducing the number of biopsies performed.

The primary goal of this study was to develop sensitive, specific and reliable assays to quantitate the biomarkers FLNA, FLNB and Keratin-19 in serum and evaluate their clinical utility. The identification of biomarker panels for health problems such as cancer are being used more frequently to address the need to better classify disease groups, predict the effect of therapeutic intervention, and monitor and detect cancer as early as possible [21]. Biomarkers are most often identified as multiple protein panels [22], which then must be verified and validated. Biomarkers can be identified in the low $\mathrm{ng} / \mathrm{ml}$ range in an MRM multiplex assay, which minimizes assay time and sample volume required [23].

This study addresses the development and clinical validation of a novel biomarker panel for improving the detection of prostate cancer. A recent study by this group discovered three novel biomarkers, FLNA, FLNB and KRT19 for prostate cancer $[13,24]$. The Prostate Cancer Biomarker proteins in the panel have been shown in previous studies to have links to cancer. Previous work has shown the absence of KRT19 in prostate cancer cells compared to the levels observed in androgen refractory cell lines. This is suggestive of the utility of KRT19 as a biomarker for differentiating aggressive, metastatic forms of PrCa. Additionally, altered levels of KRT19 expression have been demonstrated in the bone marrow of metastatic PrCa patients [25]. FLNA and FLNB belong to a family of large actin-binding filamins and play a major role in cell migration, vascular development, extracellular signaling, and activity of integrins [26]. FLNA has been described previously as being involved in normal prostate physiology and in $\mathrm{PrCa}$ metastases [26-28]. FLNB was also shown to be involved in tumor growth and metastases [29].

New assays were developed for FLNA (ELISA and IPMRM) and FLNB (ELISA). The IPMRM assay was especially suited for detection of FLNA in serum, which is more abundant and has been detected in cleaved fragments [30]. ELISA alone may not detect all forms of FLNA in a serum sample. IPMRM allows detection of different peptides along the length of the entire protein. IPMRM assays were not developed for FLNB and KRT19 as both proteins were low in abundance in the serum samples.

In this study, over 500 serum samples were screened against the PrCa Biomarker Panel (FLNA, FLNB, KRT19), and this data was combined with age, PSA test results and Gleason score to assess whether this combinatorial approach was better at predicting prostate cancer, high-risk disease versus indolent disease and discriminating between benign prostatic hyperplasia and cancer than the PSA test alone. Data was analyzed by regression modelling. The PrCa biomarkers FLNA, FLNB, age and PSA predicted the likelihood of a patient having prostate cancer better than PSA alone. This was an improvement over the standard PSA test, and could reduce the number of unnecessary biopsies in this population.

The biomarker panel FLNB, age and PSA showed improved sensitivity and specificity over the use of PSA alone in predicting whether patients had Gleason score $\geq 7$ or lower Gleason score $\leq 6$. Additionally, the biomarkers FLNA, KRT19 and age were able to improve the classification of whether patients had benign prostatic hyperplasia or cancer over the PSA test alone. Current commercially available tests include the $4 \mathrm{~K}$ score [31], which has been extensively tested in Europe and the US, and discriminates between high-risk and low-risk disease. This test utilizes the four-kallikrein (KLK) panel immunoassay of KLK2, total PSA, intact PSA, and free PSA in combination with a patient's age, DRE results and prior biopsy status. This information is analyzed by an algorithm to determine the percentage risk for aggressive prostate cancer. 4Kscore test was used in a prospective validation study in the US in 2014, and performed well in identifying patients with high risk disease. However, this test still requires a prostate biopsy and is heavily dependent on PSA levels. In 
Citation: Ravipaty S, Wu W, Dalvi A, Tanna N, Andreazi J, et al. (2017) Clinical Validation of a Serum Protein Panel (FLNA, FLNB and KRT19) for Diagnosis of Prostate Cancer. J Mol Biomark Diagn 8: 323. doi: 10.4172/2155-9929.1000323

comparison, the test described in this manuscript with FLNA, FLNB and KRT19, does not require invasive procedures to be performed on patients, and is also able to distinguish between benign prostatic hyperplasia and prostate cancer. The PHI (prostate health index) test also discriminates between high and low risk cancers. PHI was shown in a European study to be more accurate than PSA alone in predicting prostate cancer in obese patients [32]. However, it is unclear at present if PHI can discriminate between intermediate PSA values [33-35].

Prostate cancer antigen 3 (PCA3) is a non-PSA-based test of the expression of long non-coding RNA that is elevated in over $90 \%$ of PrCa tissue, but is not found in BPH or healthy tissues [36]. This is a non-invasive urine test that in combination with PSA improves PrCa prediction [37]. The androgen-induced transmembrane protease, serine 2 (TMPRSS2-ERG) is detected in urine samples of suspected PrCa patients. However, TMPRSS2-ERG is absent in $50 \%$ of cancers, and therefore it must be multiplexed with other biomarkers such as PCA3 [38]. In a study of 1300 men combination testing with TMPRSS2$E R G$ and PCA3 improved the sensitivity of PrCa diagnosis [39]. However, both PCA3 and TMPRSS2-ERG are dependent on relative PSA expression diagnosis [39,38]. The biomarker alpha-methylacylCoA racemase $(A M A C R)$, detected by RNA expression profiling demonstrates high sensitivity and specificity in prostate biopsy tissue [40]. However this biomarker is not specific to prostate cancer, nor can it be used for detection of invasive cancer in urine [41], but can be used when prostate biopsy analysis is ambiguous [42]. In conclusion, the Prostate Cancer Biomarker Panel developed in this study demonstrates an advantage over existing tests in that it not only discriminates between high and low-risk disease, it also discriminates between cancer and benign prostatic hyperplasia. Use of these biomarkers will potentially allow for more accurate diagnostic and treatment decisions, and improve the accuracy of disease prognosis by better distinguishing between indolent and high-risk disease.

\section{Acknowledgements}

The authors would like to thank Drs. Khampaseuth Thapa and Kim Wilkinson for writing and editing assistance, and Jiaqi Qu for help with data analysis. This study was funded in its entirety by BERG, LLC and National Cancer Institute R01 DK065977.

\section{Conflict of Interest}

SR, WW, AD, NT, JA, TF, AK, CL, JG, SS, PT, MK, VV, RS, NN and VA are current or previous employees of BERG, LLC and have stock. NN is also co-founder of BERG, LLC. EK and ED are consultants for BERG, LLC. The views expressed in this manuscript are those of the authors and do not reflect the official policy of the Department of the Army, Department of Defense or the U.S. government.

\section{References}

1. Siegel RL, Miller KD, Jemal A (2016) Cancer statistics, 2016. CA Cancer J Clin 66: 7-30.

2. Gardiner RACS, Williams SG, Yaxley J, Samaratunga H, Frydenberg M (2014) Prostate cancer part one: Detection. Endotext, South Dartmouth, MA.

3. Alberts AR, Schoots IG, Roobol MJ (2015) Prostate-specific antigen-based prostate cancer screening: Past and future. Int J Urol 22: 524-532.

4. Moyer VA; Force USPST (2012) Screening for prostate cancer: US Preventive services task force recommendation statement. Ann Intern Med 157: 120-134.

5. Gudmundsson J, Besenbacher S, Sulem P, Gudbjartsson DF, Olafsson I, et al (2010) Genetic correction of psa values using sequence variants associated with psa levels. Sci Transl Med 2: 62ra92.

6. Devaney JM, Wang S, Furbert-Harris P, Apprey V, Ittmann M, et al. (2015) Genome-wide differentially methylated genes in prostate cancer tissues from african-american and caucasian men. Epigenetics 10: 319-328.

7. Dhir R, Vietmeier B, Arlotti J, Acquafondata M, Landsittel D, et al. (2004) Early identification of individuals with prostate cancer in negative biopsies. J Urol 171: $1419-1423$
8. Erdmann K, Kaulke K, Thomae C, Huebner D, Sergon M, et al. (2014) Elevated expression of prostate cancer-associated genes is linked to down-regulation of micrornas. BMC Cancer 14: 1-14.

9. Tomlins SA, Day JR, Lonigro RJ, Hovelson DH, Siddiqui J, et al. (2016) Urine tmprss2: Erg plus pca3 for individualized prostate cancer risk assessment. Eur Urol 70: 45-53.

10. Cullen J, Rosner IL, Brand TC, Zhang N, Tsiatis AC, et al. (2015) A biopsybased 17-gene genomic prostate score predicts recurrence after radical prostatectomy and adverse surgical pathology in a racially diverse population of men with clinically low- and intermediate-risk prostate cancer. Eur Urol 68 123-131.

11. Gronberg H, Adolfsson J, Aly M, Nordstrom T, Wiklund P, et al. (2015) Prostate cancer screening in men aged 50-69 years (sthlm3): A prospective populationbased diagnostic study. Lancet Oncol 16: 1667-1676.

12. Liong ML, Lim CR, Yang $\mathrm{H}$, Chao S, Bong CW, et al. (2012) Blood-based biomarkers of aggressive prostate cancer. PLoS One 7: e45802.

13. Narain NR, Diers A, Ouro-Djobo R, Chan J, Rodrigues LO, et al. (2015) Abstract 538: Identification and validation of novel prostate cancer biomarkers using the berg interrogative biology ${ }^{\mathrm{TM}}$ platform. Cancer Res 75: 538-538.

14. MacLean B, Tomazela DM, Shulman N, Chambers M, Finney GL, et al. (2010) Skyline: An open source document editor for creating and analyzing targeted proteomics experiments. Bioinformatics 26: 966-968.

15. Halquist MS, Thomas Karnes H (2011) Quantitative liquid chromatography tandem mass spectrometry analysis of macromolecules using signature peptides in biological fluids. Biomed Chromatogr 25: 47-58.

16. Nicol GR, Han M, Kim J, Birse CE, Brand E, et al. (2008) Use of an immunoaffinity-mass spectrometry-based approach for the quantification of protein biomarkers from serum samples of lung cancer patients. Molecular \& Cellular Proteomics 7: 1974-1982.

17. Kulasingam V, Smith CR, Batruch I, Buckler A, Jeffery DA, et al. (2008) "Product ion monitoring" assay for prostate-specific antigen in serum using a linear ion-trap. J Proteome Res 7: 640-647.

18. Berna M, Ackermann B (2009) Increased throughput for low-abundance protein biomarker verification by liquid chromatography/tandem mass spectrometry Anal Chem 81: 3950-3956.

19. Klein T, Palisaar RJ, Holz A, Brock M, Noldus J, et al. (2010) The impact of prostate biopsy and periprostatic nerve block on erectile and voiding function: A prospective study. J Urol 184: 1447-1452

20. Ma X, Wang R, Long JB, Ross JS, Soulos PR, et al. (2014) The cost implications of prostate cancer screening in the medicare population. Cancer 120: 96-102.

21. Parker CE, Borchers $\mathrm{CH}$ (2014) Mass spectrometry based biomarker discovery, verification, and validation-quality assurance and control of protein biomarker assays. Mol Oncol 8: 840-858.

22. Anderson NL, Anderson NG (2002) The human plasma proteome: History, character, and diagnostic prospects. Mol Cell Proteomics 1: 845-867.

23. Anderson L, Hunter CL (2006) Quantitative mass spectrometric multiple reaction monitoring assays for major plasma proteins. Mol Cell Proteomics 5 : 573-588.

24. Sarangarajan RCJ, Grund E, Vishnudas VK, Luan S, Akmaev VR, et al. (2013) Abstract 7: Berg interrogative biology ${ }^{\mathrm{TM}}$ platform unravels novel biomarkers flnb and ly 9 in combination with psa enhances specificity in serum of patients with prostate cancer. Cancer Research 73: 7.

25. Guzvic M, Braun B, Ganzer R, Burger M, Nerlich M, et al. (2014) Combined genome and transcriptome analysis of single disseminated cancer cells from bone marrow of prostate cancer patients reveals unexpected transcriptomes. Cancer Res 74: 7383-7394.

26. Baldassarre M, Razinia Z, Burande CF, Lamsoul I, Lutz PG, et al. (2009) Filamins regulate cell spreading and initiation of cell migration. PLoS One 4 e7830.

27. Sun GG, Lu YF, Zhang J, Hu WN (2014) Filamin a regulates mmp-9 expression and suppresses prostate cancer cell migration and invasion. Tumour Biol 35 3819-3826.

28. Bedolla RG, Wang Y, Asuncion A, Chamie K, Siddiqui S, et al. (2009) Nuclear versus cytoplasmic localization of filamin a in prostate cancer: 
Citation: Ravipaty S, Wu W, Dalvi A, Tanna N, Andreazi J, et al. (2017) Clinical Validation of a Serum Protein Panel (FLNA, FLNB and KRT19) for Diagnosis of Prostate Cancer. J Mol Biomark Diagn 8: 323. doi: 10.4172/2155-9929.1000323

Page 8 of 8

Immunohistochemical correlation with metastases. Clin Cancer Res 15: 788-796.

29. Bandaru S, Zhou AX, Rouhi P, Zhang Y, Bergo MO, et al. (2014) Targeting filamin $b$ induces tumor growth and metastasis via enhanced activity of matrix metalloproteinase-9 and secretion of vegf-a. Oncogenesis 3: e119.

30. Liu X, Wang C, Chen Z, Jin Y, Wang Y, et al. (2011) Microrna-138 suppresses epithelial-mesenchymal transition in squamous cell carcinoma cell lines. Biochem J 440.

31. Punnen S, Pavan N, Parekh DJ (2015) Finding the wolf in sheep's clothing: The $4 \mathrm{k}$ score is a novel blood test that can accurately identify the risk of aggressive prostate cancer. Rev Urol 17: 3-13.

32. Abrate A, Lazzeri M, Lughezzani G, Buffi N, Bini V, et al. (2015) Clinical performance of the prostate health index (phi) for the prediction of prostate cancer in obese men: Data from the prometheus project, a multicentre european prospective study. BJU International 115: 537-545.

33. Lazzeri M, Haese A, de la Taille A, Palou Redorta J, McNicholas T, et al. (2013) Serum isoform [-2] propsa derivatives significantly improve prediction of prostate cancer at initial biopsy in a total psa range of 2-10 ng/ml: A multicentric european study. Eur Urol 63: 986-994.

34. Fossati N, Lazzeri M, Haese A, McNicholas T, de la Taille A, et al. (2015) Clinical performance of serum isoform [-2] propsa (p2psa), and its derivatives $\%$ p2psa and the prostate health index, in men aged <60 years: Results from a multicentric european study. BJU Int 115: 913-920.

35. Filella X, Foj L, Auge JM, Molina R, Alcover J (2014) Clinical utility of \% p2psa and prostate health index in the detection of prostate cancer. Clin Chem Lab Med 52: 1347-1355.

36. de Kok JB, Verhaegh GW, Roelofs RW, Hessels D, Kiemeney LA, et al. (2002) Dd3 (pca3): A very sensitive and specific marker to detect prostate tumors. Cancer Res 62: 2695-2698

37. Wang R, Chinnaiyan AM, Dunn RL, Wojno KJ, Wei JT (2009) Rational approach to implementation of prostate cancer antigen 3 into clinical care. Cancer 115: 3879-3886.

38. Hessels D, Smit FP, Verhaegh GW, Witjes JA, Cornel EB, et al. (2007) Detection of tmprss 2-erg fusion transcripts and prostate cancer antigen 3 in urinary sediments may improve diagnosis of prostate cancer. Clin Cancer Res 13: $5103-5108$

39. Tomlins SA, Aubin SM, Siddiqui J, Lonigro RJ, Sefton-Miller L, et al. (2011) Urine tmprss 2: Erg fusion transcript stratifies prostate cancer risk in men with elevated serum psa. Sci Transl Med 3: 94ra72.

40. Rubin MA, Zhou M, Dhanasekaran SM, Varambally S, Barrette TR, et al. (2002) Alpha-methylacyl coenzyme a racemase as a tissue biomarker for prostate cancer. Jama 287: 1662-1670.

41. Laxman B, Morris DS, Yu J, Siddiqui J, Cao J, et al. (2008) A first-generation multiplex biomarker analysis of urine for the early detection of prostate cancer Cancer Res 68: 645-649.

42. Prensner JR, Rubin MA, Wei JT, Chinnaiyan AM (2012) Beyond psa: The next generation of prostate cancer biomarkers. Sci Transl Med 4: 127 rv123. 